\section{Endoscopic Spraying of Sucralfate Using the Outer Sheath of a Clipping Device}

Sucralfate is a very useful drug for ulcer healing, since it adheres to the ulcer base $[1,2]$ and provides mucosal cytoprotection [3] in addition to having an antacid effect. The drug has a long history since it was released in 1968, but until recently formulations were limited to tablets and powder. In 1996, a liquid formulation was introduced and endoscopic application via the instrumentation channels of the endoscope became possible. It may be beneficial to administer this drug, especially after endoscopic treatment for hemorrhage from various sources. Maalox is another liquid antacid that is widely used, but it is considered to possess only a modest effect on ulcer healing in comparison with sucralfate [4]. The disadvantage of sucralfate, however, is its high viscosity, which may cause failure to spray the drug on target from the endoscope and lead to coating of the lens and loss of video. When commercial endoscopic sprayers are used, a high pressure is required for administration and only a small amount of the drug will reach the target after time-consuming effort.

Therefore, we applied the outer sheath of a clipping device (type HX-6UR-1; Olympus, Tokyo, Japan), with a maximum outer diameter of $2.8 \mathrm{~mm}$, as a thicker spray tube. The top hole of the outer sheath (intended for attachment of a coil sheath and approximately $1.1 \mathrm{~cm}$ in diameter) was covered with an original rubber cap. A 20-ml disposable syringe of sucralfate was connected to a small hole which had been designed for the purpose of washing out the sheath (Figure 1). Using this sprayer, we could administer sucralfate

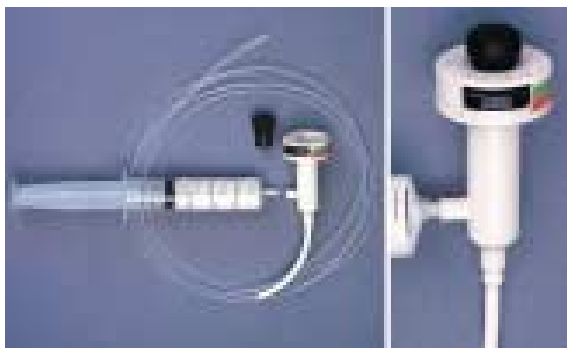

Figure 1 The outer sheath of a clipping device, with an original rubber cap and a 20-ml disposable syringe containing sucralfate. to targets easily and precisely (Figure 2 ). Although it is not proven that topical administration of sucralfate really improves the outcome, endoscopic spraying of sucralfate into the digestive tract might help to reduce the risk of rebleeding after hemostasis and to promote the healing of ulcers and tissue damage.
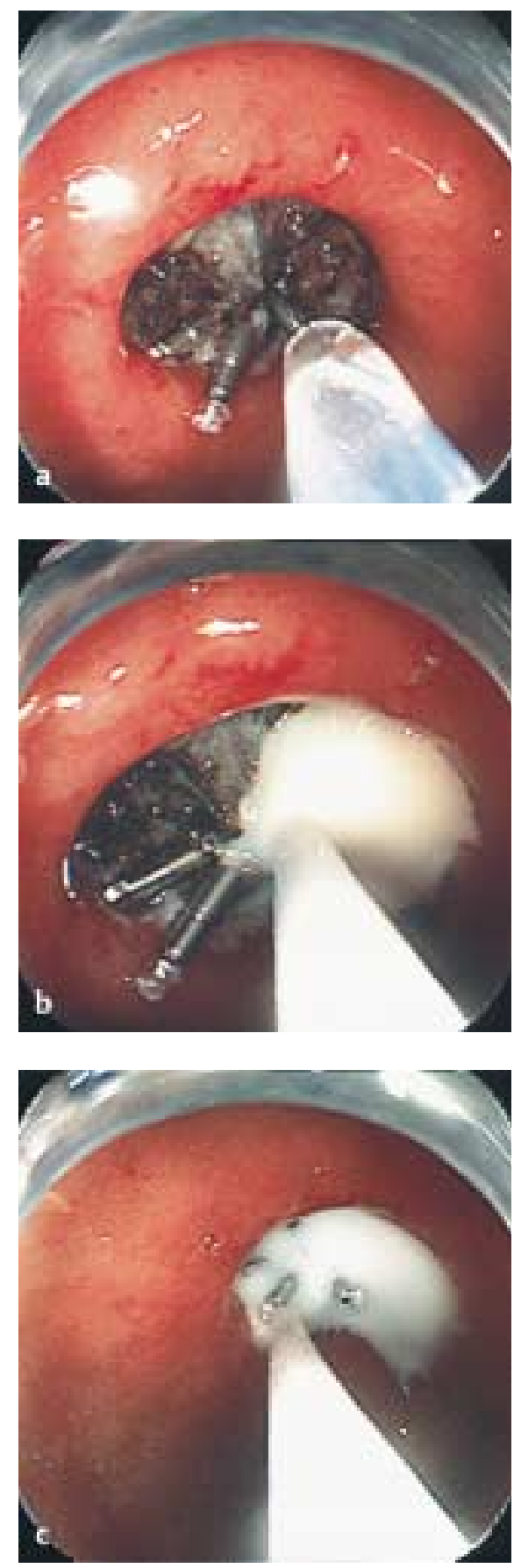

Figure 2 a-c Endoscopic spraying of sucralfate onto a gastric ulcer.
M. Fujishiro ${ }^{1}$, N. Yahagi ${ }^{1}$, M. Oka ${ }^{1}$,

S. Enomoto ${ }^{1}$, N. Yamamichi ${ }^{1}$,

N. Kakushima ${ }^{1}$, A. Tateishi ${ }^{1}$, T. Wada ${ }^{2}$, Y. Shimizu ${ }^{3}$, M. Ichinose ${ }^{3}$, T. Kawabe ${ }^{1}$, M. Omata ${ }^{1}$

${ }^{1}$ Department of Gastroenterology, Faculty of Medicine, University of Tokyo, Tokyo, Japan

2 Department of Endoscopy,

The Mutual Aid Association for Tokyo

Metropolitan, Teachers and Officials,

Sanraku Hospital Tokyo, Japan

${ }^{3}$ Second Department of Internal

Medicine, Wakayama Medical

University, Wakayama, Japan

\section{References}

${ }^{1}$ Nagashima R, Hinohara Y, Hirano T et al. Selective binding of sucralfate to ulcer lesion. II. Experiments in rats with gastric ulcer receiving ${ }^{14} \mathrm{C}$-sucralfate or potassium ${ }^{14} \mathrm{C}$-sucrose sulfate. Arzneimittelforschung 1980; 30: 84-88

${ }^{2}$ Nagashima R, Hinohara Y, Hirano T. Selective binding of sucralfate to ulcer lesion. III. Experiments in rats with duodenal ulcer receiving ${ }^{14} \mathrm{C}$-sucralfate. Arzneimittelforschung 1980; 30: $88-$ 91

${ }^{3}$ Shorrock CJ, Garner A, Hunter AH et al. Effect of bismuth subcitrate and sucralfate on rat duodenal and human gastric bicarbonate secretion in vivo. Gut 1990; 31: $26-31$

${ }^{4}$ Okabe S, Takeuchi K, Kunimi H et al. Effects of an antiulcer drug, sucralfate (a basic aluminum salt of sulfated disaccharide), on experimental gastric lesions and gastric secretion in rats. Dig Dis Sci 1983; 28: 1034 - 1042

Corresponding Author

\section{Fujishiro, M.D.}

Department of Gastroenterology Faculty of Medicine University of Tokyo 7-3-1 Hongo, Bunkyo-ku 113-8655 Tokyo Japan Fax: $\quad+81-3-58008806$ E-mail: mtfujish-kkr@umin.ac.jp 